\title{
KINETICS OF DAPAGLIFLOZIN AFTER SINGLE DOSE ORAL ADMINISTRATION OF A 10 MG IMMEDIATE RELEASE TABLET
}

\section{MONICA OROIAN ${ }^{a, b}$, ADRIANA MARCOVICI ${ }^{b}$, DIANA IOANA POP ${ }^{a, b}$, SANDEEP BHARDWAJ' ${ }^{\mathrm{b}}$, ARSHAD KHUROO', ANA-MARIA GHELDIU ${ }^{d, *}$, LAURIAN VLASE ${ }^{a}$}

\begin{abstract}
The present study aimed to elucidate and describe the basic pharmacokinetics of dapagliflozin after a single dose oral administration of a $10 \mathrm{mg}$ immediate release tablet developed by Sun Pharmaceutical Industries Limited, India. Ten competing models were created in order to analyze the experimental data obtained from the 71 subjects who were enrolled and finalized two bioequivalence clinical trials, under fasting and fed state. The studies took place at the Clinical Pharmacology and Pharmacokinetics Department of Terapia S.A. Considering the Akaike index value for a rational model discrimination, model number 8 (M8) was found to be the best that fits the experimental data. The representative pharmacokinetic model involves zero order absorption kinetics with a lag time of approximately 0.3 hours, first order systemic metabolism and elimination and two-compartmental distribution. Furthermore, by using M8, the most important pharmacokinetic parameters of dapagliflozin were determined. All calculations were performed by Phoenix WinNonlin ${ }^{\circledR}$ version 6.3 (Certara, USA).
\end{abstract}

Keywords: dapagliflozin, compartmental pharmacokinetic analysis, subjects, bioequivalence clinical trials

\footnotetext{
a University of Medicine and Pharmacy 'Iuliu Hatieganu', Faculty of Pharmacy, Department of Pharmaceutical Technology and Biopharmaceutics, 8 Victor Babes str., RO-400012, ClujNapoca, Romania

b Terapia SA - a Sun Pharma Company, Department of Clinical Pharmacology and Pharmacokinetics, 124 Fabricii str., RO-400632, Cluj-Napoca, Romania

c Clinical Pharmacology and Pharmacokinetics Department, Sun Pharma Gurugram, India

d University of Medicine and Pharmacy 'Iuliu Hatieganu', Faculty of Pharmacy, Department of Pharmaceutical Botany, 23 Marinescu str., RO-400337, Cluj-Napoca, Romania

*Corresponding author: anamaria.gheldiu@yahoo.com
} 


\section{INTRODUCTION}

Dapagliflozin (see Figure 1) is an inhibitor of the human sodiumglucose co-transporter 2 (SGLT2), which is present in the proximal tubule and is responsible for the glucose reabsorption [1]. By inhibiting the SGLT2, the glucose reabsorption can be reduced while urinary excretion of the glucose is increased [2].

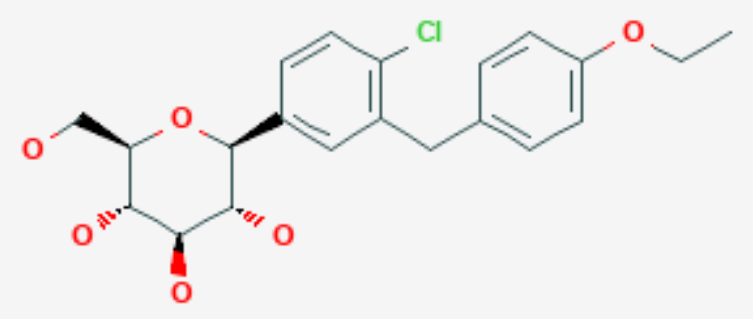

Figure 1. Chemical structure of dapagliflozin (IUPAC name: $(2 S, 3 R, 4 R, 5 S, 6 R)-2-\{4-$ chloro-3-[(4-ethoxyphenyl)methyl]phenyl\}-6-(hydroxymethyl)oxane-3,4,5-triol) [14]

Dapagliflozin was demonstrated to improve glycemic control and to prevent administration of increased doses of insulin in patients with type 2 diabetes mellitus (T2DM) while also helping them lose weight, when diet and exercise alone do not provide adequate response and when metformin administration is considered inappropriate due to intolerance $[3,4]$. Dapagliflozin is prescribed as monotherapy or associated with other oral antidiabetes substances in order to assure an improved glycemic control in patients with T2DM [5].

Dapagliflozin is metabolized via the enzyme UGT1A9 (uridine diphosphate glucuronosyltransferase 1A9), present in the liver and kidneys, to dapagliflozin 3-O-glucuronide. Its metabolite is devoid of pharmacological activity [6].

Compartmental pharmacokinetic analysis offers a better understanding of the relationship between the pharmacological effect and the administered dose. This mathematical approach deals with the quantitative analysis of the pharmacokinetic (PK) processes that occur in the human body after drug administration until its irreversible removal from the body, namely it describes the following processes: absorption, distribution, metabolism, and elimination (ADME) [7,8]. After the active drug substance/active pharmaceutical ingredient (API) is released from the pharmaceutical formulation, it is molecularly dispersed in the internal aqueous medium (or dissolved) and then is absorbed by crossing through various biological membranes, eventually reaching the blood stream [9]. From this site, which is named the central compartment and consists of highly 298 
hydrophilic tissues and organs in addition to the blood stream, the API can be distributed to peripheral compartments, which is formed by the organs and tissues with lipophilic character. Thus, the API is exchanged both-ways in between these compartments (central and peripheral) in accordance with its chemical affinities [9]. The API can also suffer metabolism prior to elimination, process that usually inactivates the API and leads to formation of more polar metabolites, which are easier to eliminate. The process of elimination always takes place from the central compartmental and can occur by metabolism and/or excretion. Therefore, if the API undergoes distribution, it then has to return to the central compartment to be further irreversible removed from the body by the process of elimination [10]. All the aforementioned kinetic processes can be characterized by transfer rate constants. Moreover, each compartment is described by a system of differential equations for which the constant coefficients are the PK parameters. By using specific PK software, the differential equations are solved for each compartment and the values for the PK parameters are determined [10].

The knowledge of drug kinetics in the body (namely the kinetic model) is important for predicting the drug plasma levels after different doses or after multiple-dose administration. The chosen kinetic model can further be used for PK population modelling, therapeutic drug monitoring, mathematical correlations between drug kinetics and the intensity of the pharmacological effect. It also gives a deeper insight of drug interactions with other drugs administered concomitantly or with food intake. Likewise, it permits a better calculation and choice of dosage regimen for a drug in particular situations, when the PK processes of absorption, distribution, metabolism and elimination are modified consequently to disease state or altered physiology $[7,8]$.

The aim of this study was to create and use a PK model that can accurately describe the kinetic processes involved in dapagliflozin's disposition in the body (ADME), after oral administration of a single dose $(10 \mathrm{mg})$ dapagliflozin in healthy Caucasian subjects. This was assayed by comparing the predicted values with actual experimental data, obtained from two bioequivalence clinical trials performed at the Clinical Pharmacology and Pharmacokinetics Department of Terapia S.A., under fasting and fed state of the subjects.

\section{RESULTS AND DISCUSSION}

A number of 10 distinct mathematical models were created in order to evaluate the PK of dapagliflozin. The characteristics for each kinetic model are given in Table 1. 
Table 1. Pharmacokinetic models for dapagliflozin used in compartmental analysis

\begin{tabular}{|c|c|c|c|}
\hline $\begin{array}{c}\text { Pharmacokinetic } \\
\text { model }\end{array}$ & Absorption kinetics & Lag time & $\begin{array}{c}\text { Number of } \\
\text { compartment }\end{array}$ \\
\hline$M 1$ & $1^{\text {st }}$ order & No & 1 \\
\hline$M 2$ & $1^{\text {st }}$ order & Yes & 1 \\
\hline$M 3$ & Zero order & No & 1 \\
\hline$M 4$ & Zero order & Yes & 1 \\
\hline$M 5$ & $1^{\text {st }}$ order & No & 2 \\
\hline$M 6$ & $1^{\text {st }}$ order & Yes & 2 \\
\hline$M 7$ & Zero order & No & 2 \\
\hline$M 8$ & Zero order & Yes & 2 \\
\hline$M 9$ & $1^{\text {st }}$ order and zero order & Yes & 1 \\
\hline$M 10$ & $1^{\text {st }}$ order and zero order & Yes & 2 \\
\hline \multicolumn{2}{|l}{} \\
\end{tabular}

The differences between the evaluated mathematical models consisted in suppositions about the kinetic order for the process of absorption (zero order or $1^{\text {st }}$ order), the existence of the lag time prior to absorption, and the number of compartments for dapagliflozin. For instance, the first PK model (M1) assumes absorption follows a $1^{\text {st }}$ order kinetics without lag time and monocompartmental distribution for dapagliflozin. The M10 model employs the existence of a mixed order absorption process (zero order and $1^{\text {st }}$ order), with lag time, and a bicompartmental distribution. For each tested model, the processes of metabolism and elimination were regarded as $1^{\text {st }}$ order kinetic processes.

The schematic representation of the kinetic processes from model M8 are depicted in Figure 2.

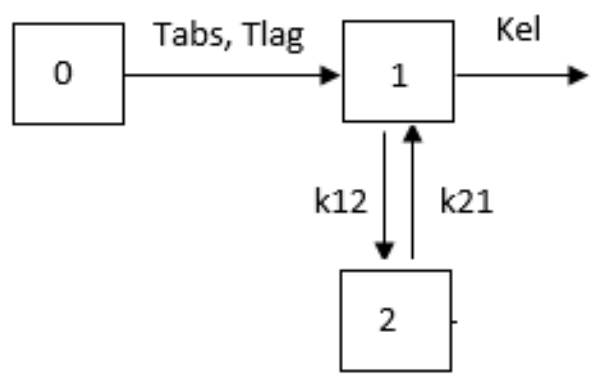

Figure 2. Schematic representation of kinetic processes for model M8, where " 0 " is absorption compartment of dapagliflozin; " 1 " is the central compartment of dapagliflozin, while "2" is the peripheral compartment; $T_{\text {lag }}$ is the latency time for absorption; $T_{\text {abs }}$ is the time needed for zero order absorption of dapagliflozin; $\mathbf{k}_{12}$ and $\mathrm{k}_{21}$ are the distribution rate constants; $\mathrm{K}_{\mathrm{el}}$ is the the elimination rate constant for dapagliflozin. The kinetic processes are depicted as straight arrows. 
For each kinetic model, the corresponding mathematical differential system of equations were written and run by the software Phoenix WinNonlin ${ }^{\circledR}$, version 6.3 (Certara, SUA). The equations for model M8 are shown in Figure 3.

M8

$$
\left\{\begin{array}{c}
\frac{\partial Q a b s}{\partial t}=-\frac{Q a b s}{T a b s} \\
\frac{\partial Q c}{\partial t}=\frac{Q a b s}{T a b s}-k e l * Q c-k 12 * Q c+k 21 * Q p \\
\frac{\partial Q c}{\partial t}=\frac{Q a b s}{T a b s}-k e l * Q c-k 12 * Q c+k 21 * Q p \\
\text { ConcDc }=\frac{Q c}{V_{-} F}
\end{array}\right.
$$

Figure 3. The mathematical equations of kinetic model $M 8$, where $Q_{a b s}$ is the amount of dapagliflozin at the site of absorption; $Q_{c}$ and $Q_{p}$ are the amount of dapagliflozin in the central and peripheral compartment, respectively; ConcDc is the plasma concentration of dapagliflozin; $Q_{a b s} / T_{a b s}$ is the rate constant of the zero order kinetic absorption; $V_{-} F$ is the apparent volume of distribution of the central compartment. The other parameters were previously described.

The ten kinetic models previously presented were implemented in Phoenix WinNonlin ${ }^{\circledR}$ software and were further used to evaluate the mean plasma concentrations versus time of dapagliflozin. For each model analysis, the settings of the software minimization engine were the following: the chosen weighting scheme was $1 / y$ (1/observed concentration), minimization method used was Gauss-Newton (Levenberg and Hartley variant), and the convergence criterion was set at 0.0001 .

For rational discrimination between the assessed kinetic models, the Akaike index value (AIC) was elected. This index considers the number of observations and number of parameters of the specific model and evaluates the goodness of fit between experimental and predicted values. The analysis software automatically calculated it and the model that best fit the experimental data was characterised by a smaller AIC value [11].

The first set of data for which was assessed the PK profile of dapagliflozin was obtained in a bioequivalence study conducted under the fasting state. Likewise, the second set of data corresponded to the clinical data of the bioequivalence test performed under the fed state of the subjects. 
Therefore, the AIC values for the ten evaluated models are presented in Figure 4 (fasting state), respectively Figure 5 (fed state).

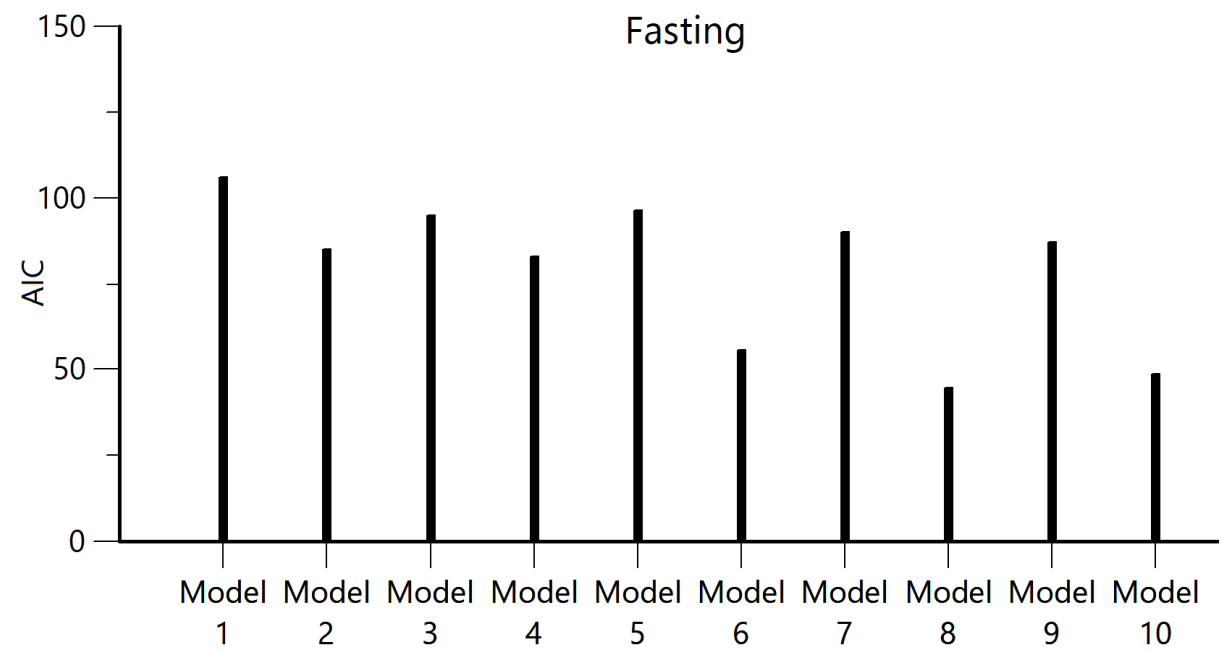

Figure 4. Akaike index values (AIC) for kinetic models used for characterization of dapagliflozin pharmacokinetics from data obtained in bioequivalence study under fasting state of subjects

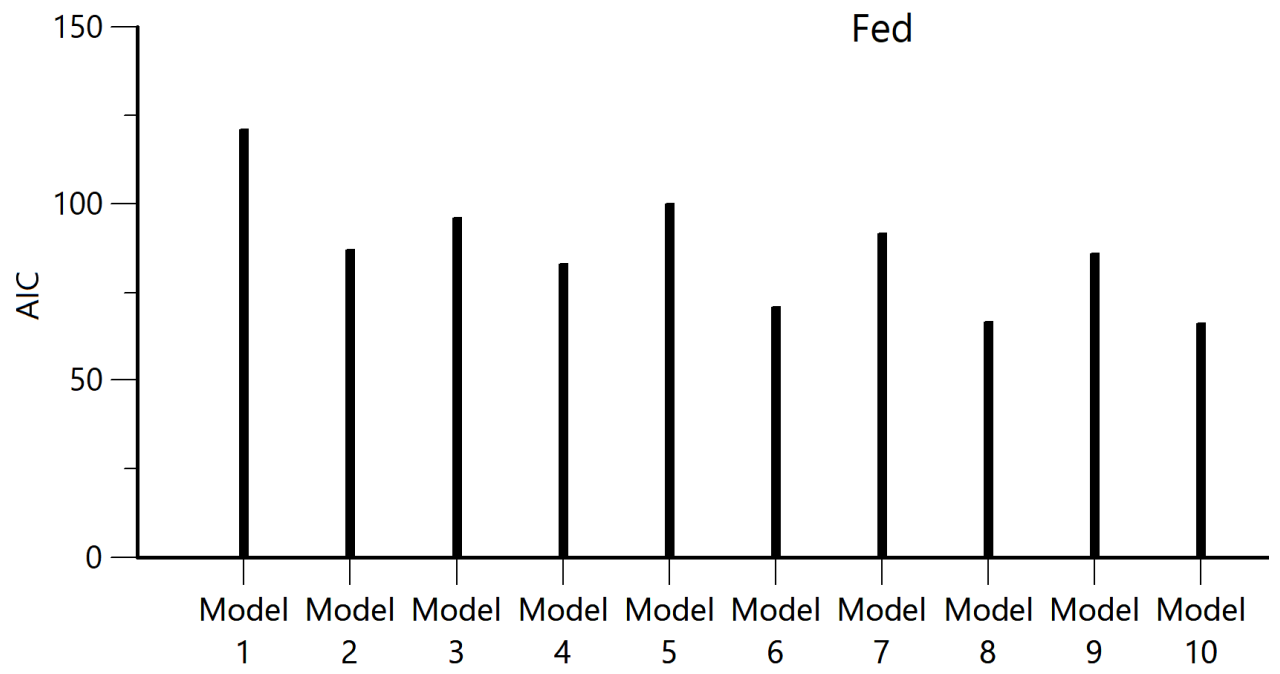

Figure 5. Akaike index values (AIC) for kinetic models used for characterization of dapagliflozin pharmacokinetics from data obtained in bioequivalence study under fed state of subjects 
By visual inspection of the AIC values presented in figures 4 and 5 , it can be concluded that model M8 fits the experimental data from both clinical trials better than its concurrent models. For this model were obtained the smallest AIC values and it was further selected as representative for the description of dapagliflozin disposition in the body after oral administration of a single dose of $10 \mathrm{mg}$ immediate release tablet.

In figure 6 is presented the typical fitting for subject 1 dataset to representative model M8, for both clinical trials, under fasting and fed state. Similar good fitting were obtained for the other 70 datasets (data not shown).
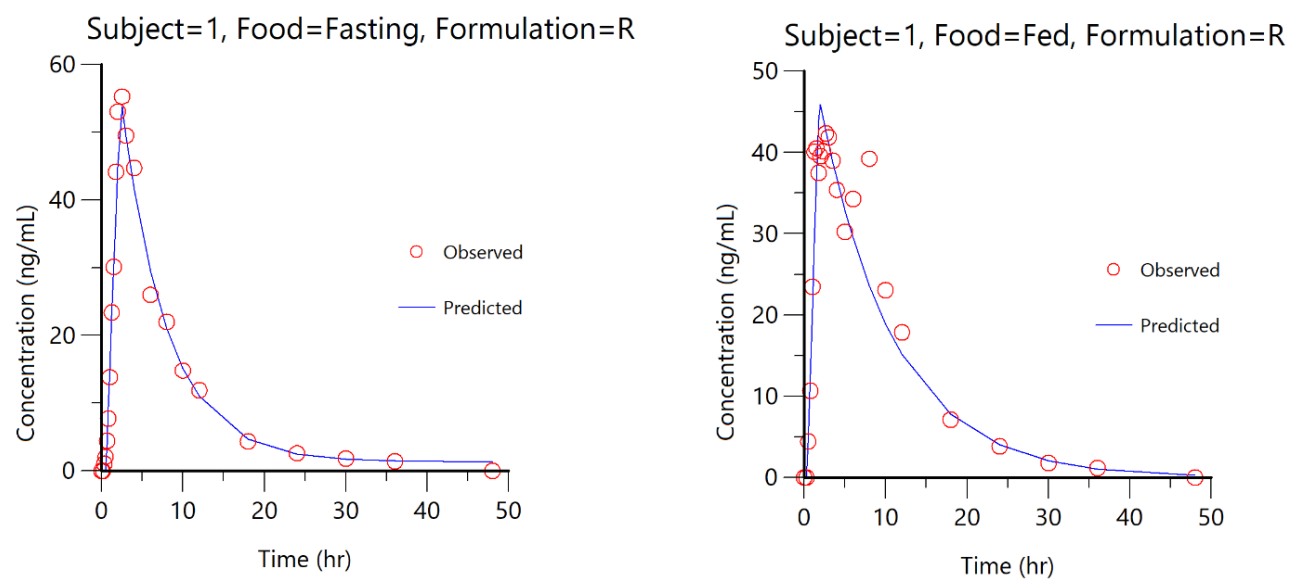

Figure 6. Typical fitting of kinetic model M8 to subject 1 dataset, for fasting and fed state of the subject in the bioequivalence clinical trial ( $\circ$ represents the experimental/observed plasma drug concentrations of dapagliflozin, the blue line represents the computed concentrations predicted by model M8)

According to kinetic model M8, the PK of dapagliflozin is characterized by a zero order absorption kinetics with lag time, bicompartmental distribution, and first order kinetics of elimination. By using this representative PK model for dapagliflozin, its characteristic PK parameters were further determined and are shown in Table 2. In the below table are summarized the main PK parameters calculated for datasets obtained in both clinical trials, under fasting and fed state of subjects, and the statistical evaluation of the differences performed with ANOVA test. 
MONICA OROIAN, ADRIANA MARCOVICI, DIANA IOANA POP, SANDEEP BHARDWAJ, ARSHAD KHUROO, ANA-MARIA GHELDIU, LAURIAN VLASE

Table 2. The kinetic parameters of dapagliflozin calculated with model M8 and the statistical evaluation of the differences between clinical trials

\begin{tabular}{|c|c|c|c|c|c|c|c|}
\hline \multirow{3}{*}{$\begin{array}{l}\text { Variable } \\
\text { (units) }\end{array}$} & \multicolumn{7}{|l|}{ Food } \\
\hline & \multicolumn{3}{|c|}{ Fasting } & \multicolumn{3}{|l|}{ Fed } & \multirow{2}{*}{$\begin{array}{l}p \text { value }^{3} \\
\text { (ANOVA) }\end{array}$} \\
\hline & Mean & $\mathrm{SD}^{1}$ & $\mathrm{CV} \%^{2}$ & Mean & SD & $\begin{array}{l}\text { CV } \\
\%\end{array}$ & \\
\hline $\mathrm{T}_{\text {lag }}(\mathrm{hr})$ & 0.290 & 0.102 & 35.2 & 0.342 & 0.268 & 78.3 & 0.949 \\
\hline $\mathrm{T}_{\mathrm{abs}}(\mathrm{hr})$ & 1.181 & 0.645 & 54.6 & 3.229 & 2.537 & 78.6 & 0.001 \\
\hline $\mathrm{K}_{12}\left(\mathrm{hr}^{-1}\right)$ & 0.088 & 0.054 & 60.9 & 0.144 & 0.237 & $\begin{array}{l}164 . \\
1\end{array}$ & 0.142 \\
\hline $\mathrm{K}_{21}\left(\mathrm{hr}^{-1}\right)$ & 0.063 & 0.042 & 66.4 & 0.118 & 0.226 & $\begin{array}{l}191 . \\
8\end{array}$ & 0.952 \\
\hline $\mathrm{K}_{\mathrm{el}}\left(\mathrm{hr}{ }^{-1}\right)$ & 0.189 & 0.072 & 38.2 & 0.162 & 0.100 & 61.8 & 0.010 \\
\hline $\begin{array}{l}\text { Vd_F } \\
\text { (L) }\end{array}$ & $\begin{array}{l}110.6 \\
85\end{array}$ & $\begin{array}{l}28.41 \\
5\end{array}$ & 25.7 & $\begin{array}{l}147.54 \\
2\end{array}$ & $\begin{array}{l}71.80 \\
2\end{array}$ & 48.7 & 0.005 \\
\hline
\end{tabular}

${ }^{1} \mathrm{SD}$ - standard deviation; ${ }^{2} \mathrm{CV} \%$ - coefficient of variation; ${ }^{3} p$ value $<0.05$ was considered statistically significant.

Dapagliflozin's basic PK profile was assessed from data obtained in two bioequivalence clinical trials, under fasting and fed state of subjects. For both cases, kinetic model M8 best fit the experimental data, even though the modified values of the calculated PK parameters in between trials showed that food intake influenced the rate constants of the kinetic processes that describe its disposition. More precisely, three PK parameters displayed statistically significant different values between studies. Firstly, the time needed for zero-order absorption process $\left(T_{\text {abs }}\right)$ varied from $1.181 \pm 0.645 \mathrm{hr}$ during the fasting study to $3.229 \pm 2.537 \mathrm{hr}$ for the fed study. Secondly, the rate constant of first-order elimination process $\left(\mathrm{K}_{\mathrm{el}}\right)$ was $0.189 \pm 0.072 \mathrm{hr}^{-1}$ for the fasting state, while for the fed state was $0.162 \pm 0.1 \mathrm{hr}^{-1}$. Thirdly, the apparent volume of distribution (Vd_F) was 110.685 $\pm 28.415 \mathrm{~L}$ for fasting state, $147.542 \pm 71.802 \mathrm{~L}$ for fed state, respectively.

The Vd_F for central compartment of dapagliflozin was high due to high protein binding of the drug (approximately 91\%) [12]. This PK parameter was influenced by the bioavailability of dapagliflozin after oral administration. The increase of Vd_F during the fed state study with approximately $33 \%$ was apparent, since the oral bioavailability of dapagliflozin was modified because of food intake.

It is acknowledged that food intake can influence the absorption process and the bioavailability of certain drugs [13]. In the case of dapagliflozin, the absorption onset was delayed since the lag time increased from $0.290 \pm 0.102 \mathrm{hr}$ (fasting state) to $0.342 \pm 0.268 \mathrm{hr}$ (fed state). This was 
due to an increased time necessary for gastric emptying and reach into duodenum, where the absorption begun. The time needed for the zero-order absorption process to take place registered a 2.7 -fold increase for fed state of the subjects.

According to M8, dapagliflozin displayed bi-compartmental distribution. As expected, the distribution processes from the central compartment to the peripheral compartment and reverse were not influenced by the food intake. The values for the rate constants of distribution processes $\left(K_{12}\right.$ and $K_{21}$, with $\mathrm{K}_{12}>\mathrm{K}_{21}$ ) did not register a statistically significant difference between clinical trials.

Dapagliflozin was eliminated following a first-order kinetic process, characterised by a rate constant of $0.189 \pm 0.072 \mathrm{hr}^{-1}$, under the fasting state. The intake of food modified the $\mathrm{K}_{\mathrm{el}}$ to $0.162 \pm 0.1 \mathrm{hr}^{-1}$; this decrease was caused by the modification of the bioavailability of dapagliflozin following oral administration. $\mathrm{K}_{\mathrm{el}}$ depends upon two physiological parameters: clearance in a directly proportional manner - and Vd_F - inversely proportional $[9,10]$, thus it was expected that an increase in the apparent volume of distribution (due to modified oral bioavailability) would lead to a decreased value for $\mathrm{K}_{\mathrm{el}}$. However, this alteration of the $\mathrm{K}_{\mathrm{el}}$ value lacks actual clinical importance.

The increase of the variability for the calculated PK parameters between clinical trials was due to food-effect in addition to natural biological and physiological differences between subjects (inter-subject variability).

\section{CONCLUSIONS}

Ten different mathematical models were evaluated in order to find the model that best fit the experimental data (plasma concentration versus time) of dapagliflozin obtained from two clinical trials. After data analysis, according to AIC values, the model M8 was found to best fit the data from both studies, conducted under fasting and fed state of subjects. Even though the food intake led to a modification of the values for certain calculated PK parameters, the basic PK profile of dapagliflozin was not influenced. The kinetics of dapagliflozin is characterised by zero-order absorption process after a lag time of approximately $0.3 \mathrm{hr}$. Following absorption, the drug is distributed between the central and peripheral compartment $\left(\mathrm{K}_{12}>\mathrm{K}_{21}\right)$ and is further eliminated from the body following first-order kinetic process. 


\section{EXPERIMENTAL SECTION}

Subjects: Both fasting and fed research studies were carried out in accordance with the Basic Principles defined in US 21 CFR Part 320, the ICH E6 (R1) (CPMP/ICH/135/95) 'Guideline for Good Clinical Practice' and the principles of the Declaration of Helsinki. The protocols of the studies were approved by National Agency for Medicines and Medical Devices, Romania and the Bioethics National Committee of the Medicines and Medical Devices, Romania. After the subjects gave their written informed consent for participation in the study, the investigators performed the screening procedures and subjects further included in studies were declared healthy and filling all inclusion criteria of the studies.

The study was conducted in the Clinical Unit of the Clinical Pharmacology and Pharmacokinetics Department of Terapia SA, Romania.

The studies were design as open label, balanced, randomized, twoperiod, two-sequence, single-dose, crossover study in fasting condition and under fed condition, respectively.

Up-to-date, no differences regarding the PK profile between genders were reported in the scientific literature, furthermore no gender effect was observed in the bioequivalence studies conducted by Clinical Unit of the Clinical Pharmacology and Pharmacokinetics Department of Terapia SA, Romania. Consequently, the subjects were of mixed gender, women and men, respectively.

Sample size estimation for both studies was based on available in-house study data on Dapagliflozin tablets. 48 Caucasian subjects were enrolled in the fasting study planned based on the assumptions that Test/Reference ratio is in the range of $90-110 \%$, an expected intra-subject CV of approximately $20 \%$, power of $80 \%$ and possible dropouts and/or withdrawals. A number of 38 subjects finalized the fasting study, of which 12 were women and 26 were men. Considering a Test/Reference ratio of $95-105 \%$ and intra-subject CV of approximately $24 \%$ and a power of $90 \%$ to show bioequivalence under bioequivalence assumptions and to allow for possible dropouts and/or withdrawals, 44 subjects were considered to be included in the fed study out of which 33 subjects finalized ( 9 women and 24 men). Thus, from the 92 subjects initially enrolled in both bioequivalence study, 71 subjects finalized the studies (21 women and 50 men). Their datasets were further used for PK modeling assay $(n=71)$.

\section{Study protocol}

Fasting study: After an overnight fasting state of at least 10 hours, the subjects were administered the drug product containing dapagliflozin $10 \mathrm{mg}$ with $240 \mathrm{~mL}$ of a $20 \%$ glucose solution in water at ambient temperature. Approximately $60 \mathrm{~mL}$ of $20 \%$ glucose solution was administered every 15 
minutes for up to 4 hours post dosing to prevent hypoglycemia. During housing subjects received standard meals, identical for both periods. For complete elimination of dapagliflozin from the body, the wash-out between study periods was 7-days.

The blood samples were collected predose and at $0.16,0.33,0.5$, $0.67,0.83,1,1.25,1.5,1.75,2,2.5,3,4,6,8,10,12,18,24,30,36$ and 48 hours post-dose in each period of the study

Fed study: Following fasting period of at least 10 hours, the subjects started the recommended high-fat high-calorie standard meal, 30 minutes prior to administration of the drug product. The drug product was administered with $240 \mathrm{~mL}$ of $20 \%$ glucose solution. Approximately $60 \mathrm{~mL}$ of $20 \%$ glucose solution was administered in approximately every 15 minutes for up to 4 hours post dose. During housing subjects received standard meals, identical for both periods. The wash-out between study periods was 7-days.

Blood samples were collected predose and at $0.25,0.5,0.75,1,1.25$, $1.5,1.75,2,2.33,2.67,3,3.5,4,5,6,8,10,12,18,24,30,36$ and 48 hours post-dose in each period of the study.

For both studies drinking water was not allowed for 1 hour before and after dosing. Thereafter, it was allowed at all times. Also, no food was allowed for 4 hours post dose.

Drug analysis from plasma: All samples were collected and processed under low light condition. The samples were collected in K3EDTA vacutainers. After collection, the blood samples were maintained in ice cold water bath. The blood samples were centrifuged under cooling condition $\left(4-10^{\circ} \mathrm{C}\right)$, within 90 minutes post blood sample collection, for 15 minutes at a speed of 4000 $\mathrm{rpm}$ and a set temperature of $4^{\circ} \mathrm{C}$, to separate plasma that was maintained in ice cold water bath until storage.

Mass spectrometry was performed in the negative-ion multiple reaction-monitoring mode with $\mathrm{m} / \mathrm{z}$ transitions of $407.10 \rightarrow 329.20$ for dapagliflozin and $412.10 \rightarrow 334.10$ for internal standard, which was dapagliflozin-D5.

Analyst software version 1.6.3 was used to determine peak areas of dapagliflozin and the internal standard. Subject sample concentrations were calculated from peak area ratios.

Calibration curves were linear over plasma dapagliflozin concentration ranges of 1.01 to $352.50 \mathrm{ng} / \mathrm{mL}$.

During validation the between-run accuracy was $88.79 \%$ to $95.10 \%$, between-run precision was $0.65 \%$ to $2.49 \%$, within-run accuracy was $89.44 \%$ to $94.39 \%$ and within-run precision was $1.12 \%$ to $5.64 \%$. 
Pharmacokinetic analysis: The compartmental PK analysis was performed in order to analyse the plasma concentrations versus time of dapagliflozin for each individual dataset obtained from subjects who took part in two bioequivalence studies (71 dataset).

Ten distinct kinetic models were created and implemented in Phoenix WinNonlin ${ }^{\circledR}$ software, version 6.3 (Certara, SUA) in order to assess the basic PK profile of dapagliflozin (see Table 1).

\section{ACKNOWLEDGMENTS}

Financial disclosures: Ana-Maria Gheldiu and Laurian Vlase are full-time employees of the University of Medicine and Pharmacy "Iuliu Hatieganu", ClujNapoca, Romania.

Conflicts of interest: Monica Oroian, Adriana Marcovici, Diana loana Pop, Sandeep Bhardwaj were employees of the Sun Pharmaceutical Industries Limited, India, during the conduct of this study.

\section{REFERENCES}

1. M. Hankins, K. Tsai, J. Kim, N. Hammar, Diabetes Research and Clinical Practice, 2017, 125, 29.

2. J.F. List, J.M. Whaley, Kidney International, 2011, 79(Suppl 120), S20.

3. J. Bolinder, Ö. Ljunggren, J. Kullberg, et al., The Journal of Clinical Endocrinology and Metabolism, 2012, 97(3), 1020.

4. J.P.-H. Wilding, V. Woo, N.G. Soler, et al., Annals of Internal Medicine, 2012, $156,405$.

5. B. Vakkalagadda, M.L. Vetter, J. Rana, et al., Pharmacology Research and Perspectives, 2015, 3(6), e00201, doi: 10.1002/prp2.201.

6. S. Kasichayanula, X. Liu, M. Pe Benito, et al., British Journal of Clinical Pharmacology, 2012, 76(3), 432.

7. A.-M. Gheldiu, A. Csavdari, M. Achim, L. Vlase, I. Tomuta, D.M. Muntean, Studia UBB Chemia, 2017, LXII (2), 179.

8. A.-M. Gheldiu. D.M. Muntean, I. Cristea, I. Antonescu, R. Chira, C. Ureche, L. Vlase, Revista de chimie (Bucharest), 2016, 67(4), 702.

9. L. Shargel, A.B.C. Yu, "Applied Biopharmaceutics and Pharmacokinetics", $7^{\text {th }}$ edition, McGraw Hill Education, New York, 2016, chapter 1.

10.S.S. Jambhekar, P.J. Breen, "Basic Pharmacokinetics", $2^{\text {nd }}$ edition, Pharmaceutical Press, London, 2012, chapter 19.

11. W. Pan, Biometrics, 2001, 57, 120.

12. M.A. Saeed, P. Narendran, Drug Design, Development and Therapy, 2014, 8, 2493.

13. D. Pop, A.-M. Gheldiu, M. Oroian, A. Marcovici, S. Bhardwaj, A. Khuroo, R. Kochhar, L. Vlase, Acta Medica Marisiensis, 2018, 64(4), 161.

14. Chemical structure of dapagliflozin, available at: https://pubchem.ncbi.nlm.nih.gov/substance/135257266\#section=2D-Structure. Accessed on the $22^{\text {nd }}$ of May 2019. 